and DHA-PQ, respectively. The PCR-corrected efficacy rates were estimated at $99.8 \% ; 99.7 \%$; $99.9 \%$, at day $28 \%$ and $99.3 \%$; $99.7 \%-99.9 \%$ in PYR, ASAQ and DHA-PQ, respectively.

Conclusion The parasite clearance times were comparable among the three ACT arms of treatment and their efficacy was comparable and higher than 99\%. There was no delay in parasite clearance time (PCT $\geq 72$ hour).

\section{OC 8465 THE DEVELOPMENT OF A CONJUGATE VACCINE AGAINST GROUP B STREPTOCOCCUS: AN AFRICAN PERSPECTIVE}

Seanette Wilson*, Patrick Tippoo. The Biovac Institute, Cape Town, South Africa

\subsection{6/bmjgh-2019-EDC. 18}

Background Streptococcus agalactiae, or group B streptococcus (GBS), is a gram-positive streptococcal bacterium that is wellrecognised as one of the leading causes of infant death, particularly in the early neonatal period (the first week of life). An estimated one in five pregnant women around the world carries GBS bacteria in their gastrointestinal or genitourinary tracts and vertical transmission from colonised mothers can lead to invasive disease in their offspring. A recent study conservatively estimated that out of 410,000 GBS cases globally every year, there are at least 147000 stillbirths and infant deaths. Despite being home to only $13 \%$ of the world's population, Africa has the highest GBS disease burden, with 54\% of estimated cases and $65 \%$ of stillbirths and infant deaths.

An effective GBS vaccine, given during pregnancy, is a promising strategy to protect against GBS disease. Currently, no licensed vaccine exists to prevent it, but scientific evaluation of feasibility is favourable. The leading vaccine candidates are capsular polysaccharide-protein conjugate vaccines. Evidence suggests maternal immunisation with a safe and effective GBS vaccine may reduce the disease risk in neonates and young infants.

The Biovac Institute was established as a private-public partnership and is the only Southern African vaccine manufacturer. Located in Cape Town, South Africa, Biovac's mission is to become a leading vaccine developer and producer in South Africa to increase capacity in Africa which only has four other vaccine manufacturers.

In collaboration with PATH, an international health organisation, and other partners, Biovac is developing a multivalent conjugate vaccine against GBS. The first stage of the project involves the development of biopharmaceutical manufacturing processes and analytical tests, the preparation of clinical trial product, and execution of a first-in-human clinical trial.

This presentation will provide an overview of the project, progress to date, and the path to commercialisation.

\section{OC 8469 REVIEWING INVESTIGATIONAL PRODUCT'S QUALITY ASSURANCE DOCUMENTATION IN MAJOR CLINICAL TRIAL REGISTRIES FOR POST-MARKETING CLINICAL TRIAL STUDIES}

\footnotetext{
1,2Joachim Doua, ${ }^{1,3}$ Hanneke Dominicus*. ${ }^{1}$ Consortium for African Regulatory Expertise Development (CARED), The Hague, The Netherlands; 'Johnson and Johnson, Brussels, Belgium; ${ }^{3}$ Dominicus Medicus Consultancy, The Hague, The Netherlands
}

Background The proven worrisome quality of medicines marketed in developing countries also affects clinical trials $(\mathrm{CTs})$ as they may be used as Investigational Medicinal Products (IMPs). By regulation, CT sponsors should assure IMP's quality and describe their quality measures in CT protocols that should be registered in a CT Registry (CTR). To check compliance with this regulation, we reviewed major CTRs to assess the availabilities of data fields on IMP quality for postmarketing CTs.

Methods Two reviewers independently assessed English versions of CTRs of International Committee of Medical Journal Editors (ICJME) and WHO platforms in July 2017. Each CTR was checked for availability of data fields on: brand name; manufacturer's name; regulatory approval status; approving regulator; manufacturer's compliance with Good Manufacturing Practices (cGMP); and quality testing (IMP appearance, impurities, microbial contamination, dosing). In case of discrepancy, consensus was sought.

Results Of 19 CTRs identified, 8 and 6 belonged to WHO and ICMJE, respectively, and 5 were equally part of both platforms. All CTRs had an 'intervention' data field to capture data on IMPs and IMP comparators. Unlike all others, the Canadian CTR used 'drug name' rather than 'intervention'. Only the EU CTR had data fields for 'manufacturer's name', 'product approval status', and 'approving authority'. None of the CTRs had data fields on 'cGMP' or 'quality testing'.

Conclusion None of the CTRs of ICMJE and ICTRP has adequate data fields to establish that the source of post-marketing IMPs is of assured quality. This is astonishing given the extensive requirements in WHO and ICMJE guidelines. The gap of quality assurance fields should be bridged by adding them to CTRs. Specifically, IMP quality testing should be conducted before, during, and after clinical trial completion. Until adoption of these measures, EU-CTR should be favoured for registration of CTs conducted in developing countries.

\section{OC 8474 ASSESSMENT OF ETHICS REVIEW FRAMEWORKS FOR HEALTH RESEARCH IN EAST AFRICA FOR PURPOSES OF HARMONISATION}

${ }^{1}$ Gibson S Kibiki, ${ }^{1}$ Novat Twungubumwe*, ${ }^{2}$ Francis Kombe, ${ }^{3}$ Carel IJsselmuiden, ${ }^{4}$ Prince N Bahati, ${ }^{4}$ Anatoli Kamali, ${ }^{1}$ Jean De Dieu Ngirabega. ${ }^{1}$ East African Health Research Commission, Burundi; ${ }^{2}$ Council on Health Research for Development, Kenya; ${ }^{3}$ Council on Health Research for Development, Geneva, Switzerland; ${ }^{4}$ International AIDS Vaccine Initiative, Nairobi, Kenya

\subsection{6/bmjgh-2019-EDC.20}

Background Capacity in health research ethics review is key in Africa, given the increase in research activities, complexity, and use of advanced technologies. Harmonising ethics review frameworks can address challenges attributable to these complexities. Establishing an effective harmonised framework that is optimum or protection of the research subjects requires assessment of review capacity.

The East African Health Research Commission commissioned a study to assess the capacity of Review Ethics Committees (RECs) in the East African Community (EAC) countries, as a step towards strengthening and harmonising the regions' capacity and review frameworks.

Methods A desktop review of documentation (national and institutional guidelines, policies and SOPs) was conducted in five EAC countries. Semi-structured questionnaires were used to collect data from key informants. Qualitative interviews 